\title{
Tailoring the shape of oxide complex nanostructures
}

B. Méndez, I. López, M. Alonso-Orts, A. Sanz, E. Nogales, et al. 


\title{
Tailoring the shape of oxide complex nanostructures
}

\author{
B. Méndez*a ${ }^{*}$ I. López ${ }^{\mathrm{a}, \mathrm{b}}$, M. Alonso-Orts ${ }^{\mathrm{a}}$, A. Sanz ${ }^{\mathrm{a}}$, E. Nogales ${ }^{\mathrm{a}}$, P. Hidalgo ${ }^{\mathrm{a}}, \mathrm{J}_{\text {. Piqueras }}^{\mathrm{a}}$ \\ ${ }^{a}$ Dept. Física de Materiales, Universidad Complutense de Madrid, Madrid 28040, Spain \\ bIstituto Nazionale di Ottica (INO)-CNR, via Carrara 1, 50019 Sesto Fiorentino FI, Italy
}

\begin{abstract}
The synthesis of complex nanostructures that combine materials and dimensionality, promises the ability to identify novel designs and architectures with enhanced properties that could be used in new devices. One of the building blocks in nanomaterials are nanowires, which offer several possibilities to get complex nanostructures. We present two kinds of morphologies based on oxide nanowires obtained by a thermal evaporation method. The common feature of both morphologies is a central oxide nanowire and, depending on the growth parameters, nanowires with either nanocrystallites or nano/microrods attached to the central wire are obtained. We have previously reported the fabrication of several single oxide nanowires and in particular, gallium oxide $\left(\beta-\mathrm{Ga}_{2} \mathrm{O}_{3}\right)$ and zinc germanate oxide $\left(\mathrm{Zn}_{2} \mathrm{GeO}_{4}\right)$ nanowires. Here we report the shape evolution of these nanowires by the suitable modification of the growth parameters. The addition of tin oxide $\left(\mathrm{SnO}_{2}\right)$ to the precursors and variation of the thermal treatments duration result in the formation of the above-mentioned complex nanostructures. Structural and chemical characterizations were performed by electron microscopy techniques and Raman spectroscopy. The results shed light on the understanding of the driving mechanisms that lead to the formation of complex oxide nanostructures.
\end{abstract}

Keywords: semiconducting oxide nanowires, gallium and tin oxide, germanates nanowires, electron microscopy, thermal evaporation method, Raman spectroscopy

\section{INTRODUCTION}

From the point of view of novel device technologies, nanowires elements can be considered as one of the main building blocks in innovative assemblies. The reason is the strong interest in going beyond planar technologies, where thin films are the main actors, and alternative designs as vertical ones where nanowires play a key role ${ }^{1}$. Nanowires offer several possibilities to get complex nanostructures, such as core-shell, branched wires, crossing wires or hierarchical structures, to name a few ${ }^{2}$. Moreover, nanowires could act as scaffolds or substrates for other nanomaterials, such as nanoparticles or ultrathin layers, what could widen the range of applications. Semiconducting oxide materials are attractive in the field of optoelectronics because of merging optical transparency and suitable electronic conductivity. In addition, their wide band gap character enables further applications in the ultraviolet range and in high power devices. Finally, their chemical stability and good mechanical properties make oxides an attractive choice for a number of applications ${ }^{3}$. In this work, we report on complex oxide nanostructures based on $\mathrm{Ga}_{2} \mathrm{O}_{3}, \mathrm{SnO}_{2}$, and $\mathrm{Zn}_{2} \mathrm{GeO}_{4}$, which have energy band gaps of $4.9 \mathrm{eV}$, $3.6 \mathrm{eV}$ and $3.3 \mathrm{eV}$, respectively.

A variety of synthesis routes to obtain oxide nanowires have been reported, being thermal evaporation method a rather simple one that enable to grow both undoped and doped materials. We have successfully fabricated several oxide nanowires by simple evaporation-solidification mechanism ${ }^{4-8}$. The key parameters that control the growth process are the temperature, the duration, the gas flow and the source materials. Any action over any of these factors may alter the final product, not only in their physical properties but also in the final morphology or architecture. In particular, suitable doping and orientation of $\mathrm{Ga}_{2} \mathrm{O}_{3}$ nanowires have been revealing as the driven mechanism to achieve complex $\mathrm{Ga}_{2} \mathrm{O}_{3} / \mathrm{SnO}_{2}$ nanostructures ${ }^{9-10}$. In this paper, we report on the shape evolution of complex nanostructures based on nanowires by suitable modification of the growth parameters. In particular, we report on heterostructures formed by $\mathrm{Ga}_{2} \mathrm{O}_{3}$ and $\mathrm{Zn}_{2} \mathrm{GeO}_{4}$ nanowires as the main axis, with either modulated diameter or with nanocrystallites attached to the axis.

*bianchi@ucm.es; phone +34 91394 4746; fax -34 91394 4547; finegroup.es

Nanotechnology VIII, edited by lon M. Tiginyanu, Proc. of SPIE Vol. 10248, 1024805

(c) 2017 SPIE · CCC code: $0277-786 X / 17 / \$ 18 \cdot$ doi: $10.1117 / 12.2264457$ 


\section{EXPERIMENTAL}

A pellet of a mixture of $\mathrm{Ga}_{2} \mathrm{O}_{3}$ and $\mathrm{SnO}_{2}$ compacted powders in a weight ratio (90\%:10\%) was used as substrate where Ga chips were placed onto it. The pellet was treated for 15 hours at $1500^{\circ} \mathrm{C}$ under an argon flow of $0.81 / \mathrm{min}$ and a huge amount of nanostructures was formed. Alternatively, a small amount of $\mathrm{Cr}$ impurities was added to the previous precursors, which results in changes in the final morphology of the $\mathrm{Ga}_{2} \mathrm{O}_{3} / \mathrm{SnO}_{2}$ nanostructures. On the other hand, we have previously reported the growth of undoped $\mathrm{Zn}_{2} \mathrm{GeO}_{4}$ nanowires by using a compacted pellet made of a mixture of $\mathrm{ZnO}$, Ge and carbon powders with a weight ratio 2:1:2 that acted as source and as substrate ${ }^{6}$. The thermal treatment was carried out at $800{ }^{\circ} \mathrm{C}$ for 8 hours. In this work, complex $\mathrm{Zn}_{2} \mathrm{GeO}_{4} / \mathrm{SnO}_{2}$ nanostructures were obtained by adding a $10 \%$ of $\mathrm{SnO}_{2}$ to the mixture $\{\mathrm{ZnO}+\mathrm{Ge}\}$ and followed the same thermal treatment as in Ref. 6 .

The morphology was analyzed in a Leica 440 Steroscan scanning electron microscope (SEM) while spatially resolved chemical composition was studied in the energy dispersive X-ray microanalysis (EDS) mode. Luminescence was analyzed both by Cathodoluminescence (CL) in the SEM and by photoluminescence (PL) in an optical confocal microscope (Horiba Jobin Ybon LabRam HR800). The confocal microscope was equipped with an ultraviolet laser $(\lambda=325 \mathrm{~nm})$ and enables as well to carry out Raman spectroscopy.

\section{RESULTS AND DISCUSSION}

Complex $\mathrm{Ga}_{2} \mathrm{O}_{3} / \mathrm{SnO}_{2}$ and $\mathrm{Zn}_{2} \mathrm{GeO}_{4} / \mathrm{SnO}_{2}$ micro- and nanostructures have been grown in a single step thermal treatment, via a vapor-solid (VS) mechanism. Figure 1a shows the SEM image and the $\{\mathrm{Ga}+\mathrm{Sn}\}$ elemental mapping of a representative complex oxide nanostructure composed by a main $\mathrm{Ga}_{2} \mathrm{O}_{3}$ nanowire with attached $\mathrm{SnO}_{2}$ particles. The $\mathrm{Ga}_{2} \mathrm{O}_{3}$ axis width is around $200 \mathrm{~nm}$ and the size of the $\mathrm{SnO}_{2}$ particles is about $1 \mu \mathrm{m}$. It is worth mentioning that $\mathrm{Ga}_{2} \mathrm{O}_{3}$ has a monoclinic lattice structure while $\mathrm{SnO}_{2}$ is of rutile phase. However, TEM measurements have revealed that a good lattice matching between both lattices ${ }^{10}$. In addition, we have explored the possibility to obtain this kind of morphology with other semiconducting oxides. $\mathrm{Zn}_{2} \mathrm{GeO}_{4}$ nanowires are obtained by using a mixture of $\mathrm{ZnO}$ :Ge:carbon in a 2:1:2 weight ratio as source and substrate ${ }^{6}$. In this case, a $10 \%$ of $\mathrm{SnO}_{2}$ was incorporated to the mixture. Figure $1 \mathrm{~b}$ shows that a similar complex structure with particles around an axis is formed. Figure 1c shows the elemental mappings for $\mathrm{Zn}, \mathrm{Ge}$ and $\mathrm{Sn}$ of a $\mathrm{Zn}_{2} \mathrm{GeO}_{4}$ nanowire decorated with $\mathrm{SnO}_{2}$ particles, which are doped with Ge and $\mathrm{Zn}$. The $\mathrm{SnO}_{2}$ particles show similar shapes and sizes in both cases with well-defined facets and a limited growth in the direction perpendicular to the central axis.
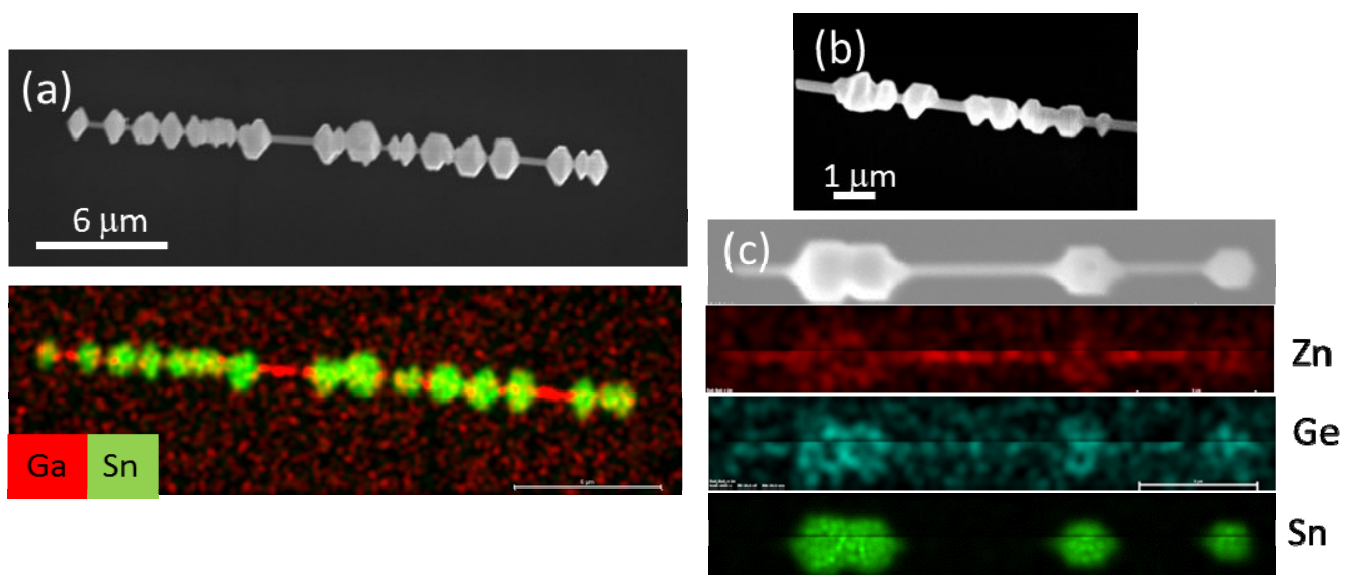

Figure 1. SEM images and elemental mappings of complex nanostructures based on a main oxide nanowire surrounded by $\mathrm{SnO}_{2}$ particles. (a) $\mathrm{Ga}_{2} \mathrm{O}_{3} / \mathrm{SnO}_{2}$ and (b-c) $\mathrm{Zn}_{2} \mathrm{GeO}_{4} / \mathrm{SnO}_{2}$ nanostructures. 

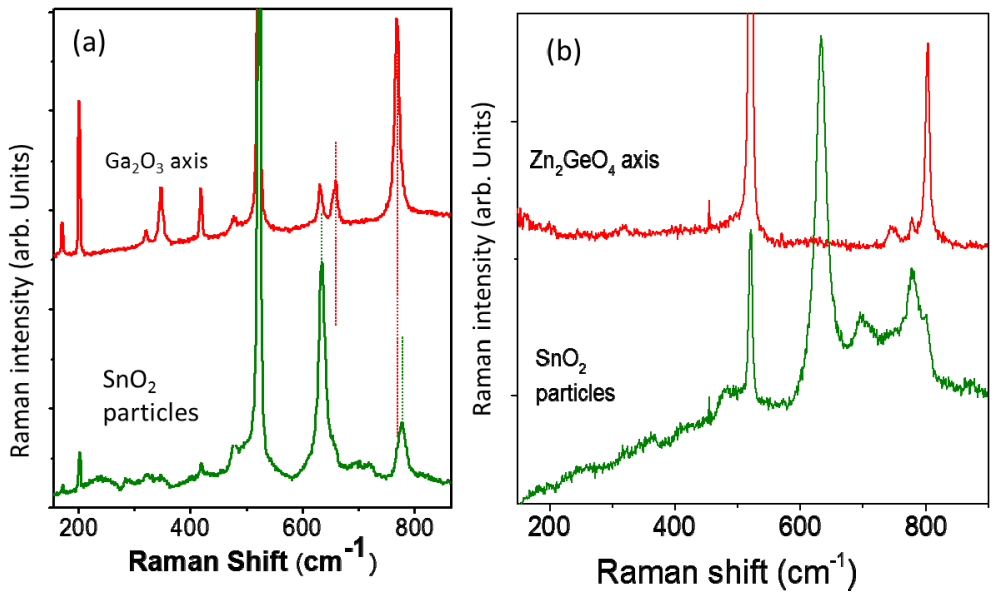

Figure 2. Raman characterization of (a) $\mathrm{Ga}_{2} \mathrm{O}_{3} / \mathrm{SnO}_{2}$ and (b) $\mathrm{Zn}_{2} \mathrm{GeO}_{4} / \mathrm{SnO}_{2}$ nanostructures.

In order to check the crystal quality of the oxide nanostructures, Raman spectra measurements have been done and summarized in Figure 2. Raman spectra from axial nanowires show a very good crystal quality in both nanostructures and characteristic modes of $\mathrm{Ga}_{2} \mathrm{O}_{3}$ and $\mathrm{Zn}_{2} \mathrm{GeO}_{4}$ are well-defined (upper curves in Figure 2). Raman spectra recorded from the attached particles are also shown (lower curves in Figure 2). $\mathrm{SnO}_{2}$ crystallites on $\mathrm{Ga}_{2} \mathrm{O}_{3}$ nanowires exhibit better crystal quality than those on $\mathrm{Zn}_{2} \mathrm{GeO}_{4}$ nanowires, which could be due to the $\mathrm{Ge}$ and $\mathrm{Zn}$ co-doping of the particles. The $520 \mathrm{~cm}^{-1}$ peak presented in all Raman spectra corresponds to the silicon substrate.

Sn impurities have been proposed as a way to increase electrical n-type conductivity in some oxides nanowires. However, this is difficult to achieve because of the impurities out-diffusion during the growth process, which is sometimes difficult to prevent. Also, the addition of Sn to the source materials have been found to influence as well the shape of oxide nanowires obtained by thermal evaporation methods, leading to branched nanowires or to nanowires with higher aspect-ratio ${ }^{4,11}$. Here, we observe that once the main $\mathrm{Ga}_{2} \mathrm{O}_{3}$ or $\mathrm{Zn}_{2} \mathrm{GeO}_{4}$ nanowires are formed, the out-diffused $\mathrm{Sn}$ impurities may be aggregated at some sites of the lateral surface of the NWs and act as nucleation centers for $\mathrm{SnO}_{2}$ particles.

Other impurity of interest in semiconducting oxides is chromium due to the intraionic red luminescence of $\mathrm{Cr}^{3+}$ cations. The quantum yield of red luminescence of $\mathrm{Cr}$ increases considerably when impurities are embedded in an oxide nanowire matrix instead of bulk material ${ }^{12}$. In a previous work on $\mathrm{Cr}$ doped $\mathrm{Ga}_{2} \mathrm{O}_{3}$ nanowires obtained after a two-step growth process, an intense red luminescence was achieved ${ }^{4}$. Also, the addition of both $\mathrm{Sn}$ and $\mathrm{Cr}$ to the precursors and a thermal treatment in a single step lead to the formation of a considerable amount of crossed $\mathrm{Ga}_{2} \mathrm{O}_{3} / \mathrm{SnO}_{2}$ nanowires ${ }^{9,10}$. These structures consist of a main $\mathrm{Ga}_{2} \mathrm{O}_{3}$ axis where a certain number of crossed $\mathrm{SnO}_{2}$ wires are grown. The formation of $\mathrm{SnO}_{2}$ particles or wires around the axis has been discussed as a function of the orientation of the axis $\mathrm{NW}^{10}$. Here we present some nanostructures that have a high number of $\mathrm{SnO}_{2}$ wires crossing the $\mathrm{Ga}_{2} \mathrm{O}_{3}$ nanowire, leading to a kind of "core-shell" morphology with a rather complex shell.
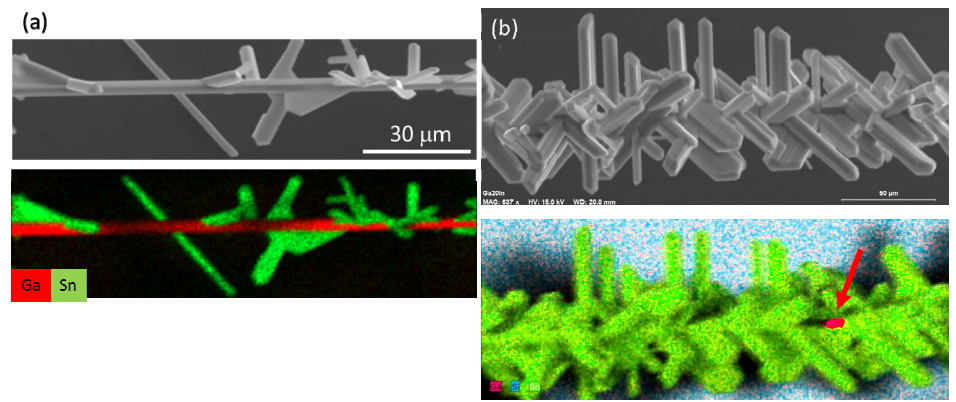

Figure 3. SEM and elemental mapping of $\mathrm{Ga}_{2} \mathrm{O}_{3}$ nanowires with different number of $\mathrm{SnO}_{2}$ crossing wires. 

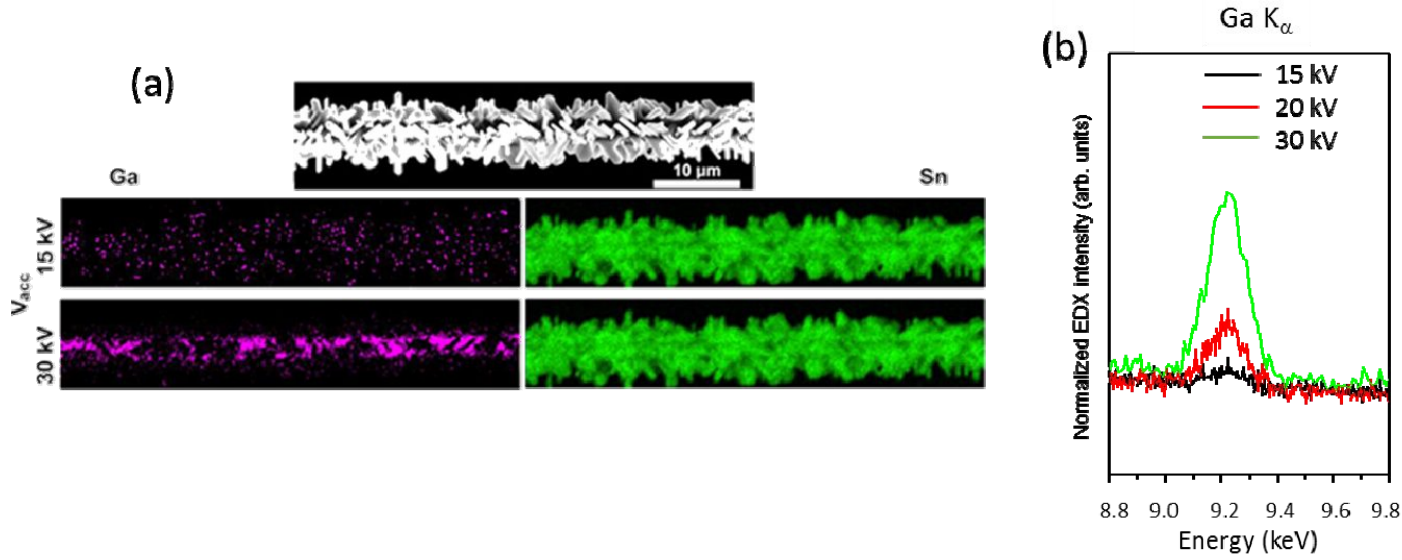

Figure 4. (a) SEM and EDX Ga and Sn mappings of complex core-shell $\mathrm{Ga}_{2} \mathrm{O}_{3} / \mathrm{SnO}_{2}$ nanostructures analyzed at $15 \mathrm{kV}$ and $30 \mathrm{kV}$ acceleration voltages. (b) Short-range energy EDX spectra showing the Ga characteristic Xray lines recorded at 15 and $30 \mathrm{kV}$.

Figure $3 \mathrm{a}$ shows a structure composed of a central $\mathrm{Ga}_{2} \mathrm{O}_{3}$ axis crossed by rather separated $\mathrm{SnO}_{2}$ wires, while Figure $3 \mathrm{~b}$ shows a more complex morphology. As the EDX mapping shows, Sn appears to cover fully the whole structure and just a short length of the axis remains uncoated (marked by an arrow in Fig. 3b). Figure 4a shows the EDX mappings for Ga and $\mathrm{Sn}$ of other of these fully coated nanowires at different acceleration voltages of the electron beam. Since the higher the energy of the electron beam, the larger the penetration depth, the results show that Ga is present at the core axis, as it can be seen in Fig. 4a (Ga mapping at $30 \mathrm{kV}$ ). Figure $4 \mathrm{~b}$ shows partial EDX spectra of the characteristic $\mathrm{K}_{\alpha}$ lines of Ga for 15,20 and $30 \mathrm{kV}$ acceleration voltages recorded from the central part of the structure. The penetration range of energetic electrons into a specimen, which beside the beam energy also depends on the material density and atomic number, can be simulated with CASINO program ${ }^{12}$. For pure $\mathrm{Ga}_{2} \mathrm{O}_{3}$, simulations of the electron paths until their thermalization yield a value of $500 \mathrm{~nm}$ for $10 \mathrm{kV}$ and about 2 microns for $25 \mathrm{kV}$. Now we have to consider as well the $\mathrm{SnO}_{2}$ shell that coats the $\mathrm{Ga}_{2} \mathrm{O}_{3}$ nanowire. We have carried out simulations by considering a $\mathrm{SnO}_{2}$ shell of several thickness (from $500 \mathrm{~nm}$ to $2000 \mathrm{~nm}$ ) surrounding the $\mathrm{Ga}_{2} \mathrm{O}_{3}$ core. The simulated profiles for electrons with energies of 15, 20 and $30 \mathrm{keV}$ impinging a $\mathrm{SnO}_{2}$ shell of $750 \mathrm{~nm}$ are shown in Figure 5. This depth is what better fits with experimental EDX results presented above (Fig.4). As it can be observed, electrons with $15 \mathrm{keV}$ test a region less than $750 \mathrm{~nm}$ in depth, but electrons with $20 \mathrm{keV}$ energy reach the $\mathrm{Ga}_{2} \mathrm{O}_{3}$ core, which would agree with the EDX signal detected in Figure 4b. These results suggest that the thickness of the $\mathrm{SnO}_{2}$ coating the $\mathrm{Ga}_{2} \mathrm{O}_{3}$ axis would be around $750-$ $1000 \mathrm{~nm}$.

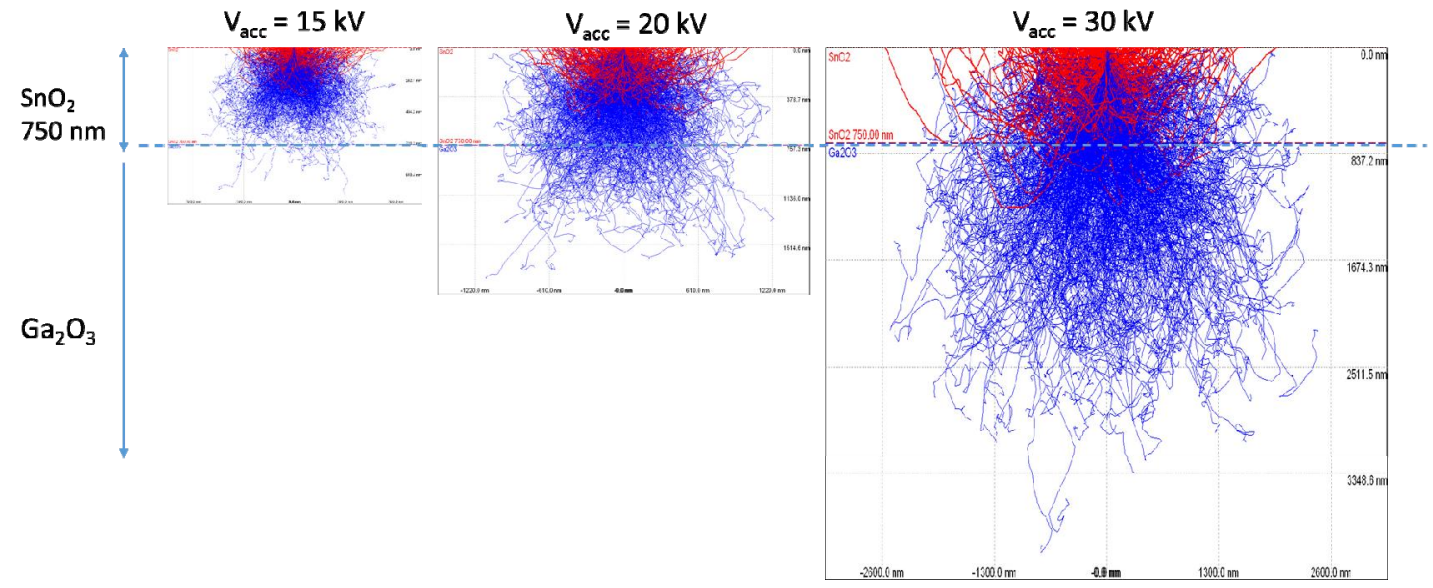

Figure 5. Montecarlo simulation (CASINO) electrons paths up until they stop (blue lines) within a sample composed of a first $\mathrm{SnO}_{2}$ layer of $750 \mathrm{~nm}$ thickness and $\mathrm{Ga}_{2} \mathrm{O}_{3}$ below. 
(a)

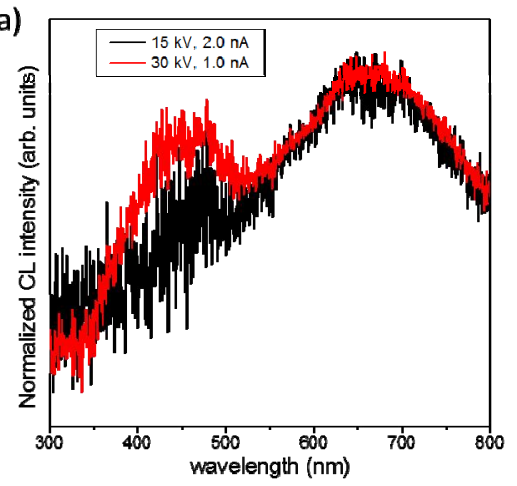

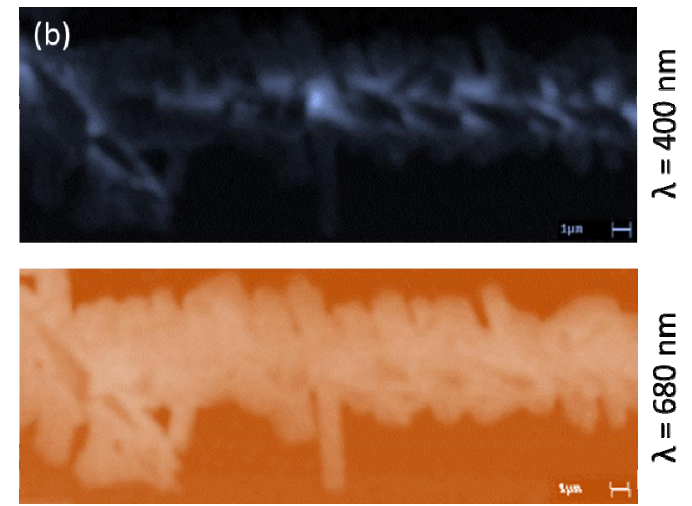

Figure 6. (a) $\mathrm{CL}$ spectra of one complex $\mathrm{Ga}_{2} \mathrm{O}_{3} / \mathrm{SnO}_{2}$ structure recorded with electrons accelerated with energies of 15 and $30 \mathrm{keV}$. (b) Monochromatic CL images at $400 \mathrm{~nm}$ (upper image) and at $680 \mathrm{~nm}$ (lower image) of one complex $\mathrm{Ga}_{2} \mathrm{O}_{3} / \mathrm{SnO}_{2}$ nanostructure, recorded at $30 \mathrm{keV}$.

One of the potential attractions of these nanostructures of mixed oxides is that different luminescent behavior is possible in the same nanostructure. We have carried out Cathodoluminescence (CL) measurements in the SEM, which enables high spatial resolution. Semiconducting oxides usually exhibit luminescence due to the radiative centers involving oxygen vacancies. Native defects in $\mathrm{Ga}_{2} \mathrm{O}_{3}$ originate a luminescence band in the UV-blue region ${ }^{14-15}$. On the other hand, $\mathrm{SnO}_{2}$ show a complex luminescence band in the green-orange-red region with several components that is affected by oxygen vacancies and surface states of the crystal facets ${ }^{16-17}$. Figure 6a shows the CL spectra of one of these complex $\mathrm{Ga}_{2} \mathrm{O}_{3} / \mathrm{SnO}_{2}$ structures (similar to that shown in Fig. 5) recorded with electron beam energies of 15 and $30 \mathrm{keV}$. The broad green band around $680 \mathrm{~nm}$, which is characteristic of $\mathrm{SnO}_{2}$, is observed in both cases, while the blue-UV band of $\mathrm{Ga}_{2} \mathrm{O}_{3}$ is only detected for $30 \mathrm{keV}$ electron beam energy due to the higher penetration depth of electrons. Figure $6 \mathrm{~b}$ shows two monochromatic CL images revealing areas emitting photons of $400 \mathrm{~nm}$ and $680 \mathrm{~nm}$ wavelengths. These selected wavelengths correspond to maxima of band emissions in $\mathrm{Ga}_{2} \mathrm{O}_{3}$ and $\mathrm{SnO}_{2}$, respectively. The images clearly reveal the spatial distribution of the luminescence centers in the complex structure.

\section{CONCLUSIONS}

We have successfully grown complex nanostructures based on oxide semiconducting materials, such as $\mathrm{Ga}_{2} \mathrm{O}_{3}, \mathrm{Zn}_{2} \mathrm{GeO}_{4}$ and $\mathrm{SnO}_{2}$, by a rather simple evaporation method. The complex structures consisted of a main oxide nanowire axis, to which either particles or rods were attached. $\mathrm{Zn}_{2} \mathrm{GeO}_{4}$ and $\mathrm{Ga}_{2} \mathrm{O}_{3}$ nanowires are the main axis and particles or rods are made of $\mathrm{SnO}_{2}$. The quality of the structure was assessed by Raman spectroscopy, which shows the characteristic peaks of the corresponding lattices. In the case of $\mathrm{SnO}_{2}$ rods grown on $\mathrm{Ga}_{2} \mathrm{O}_{3}$ nanowires, EDX microanalysis maps and CL measurements show that a core-shell structure is formed at the axis. Montecarlo simulations of the electron paths within the sample until they lose their energy agrees with a model of a shell of about $750 \mathrm{~nm}$ width for the $\mathrm{SnO}_{2}$ that coat the main $\mathrm{Ga}_{2} \mathrm{O}_{3}$ axis. These results aim to shed some light on the understanding of the driving mechanisms that lead to the formation of complex oxide nanostructures.

\section{ACKNOWLEDGMENTS}

This work has been supported by MINECO (projects CSD 2009-2013, MAT 2012 - 31959, MAT 2015 - 65274 - R FEDER). M. A-O acknowledges financial support from MEC (FPU contract). 


\section{REFERENCES}

[1] Thelander C., Agarwal P., Brongersma S., Eymery J., Feiner L.F., Forchel A., Scheffler M., Riess W., Ohlsson B.J., Gösele U., Samuelson L., "Nanowire-based one-dimensional electronics" Materials Today, 9, 28-35 (2006).

[2] Hwang Y.J., Wu C.H., Hahn C., Jeong H.E., Yang P., "Si/InGaN Core/Shell Hierarchical Nanowire Arrays and their Photoelectrochemical Properties" Nano Lett., 12, 1678-1682 (2012).

[3] Yu X., Marks T.J., Facchetti A., Nature Mater. 15, 383-396 (2016).

[4] López I., Nogales E., Méndez B., Piqueras J., Peche A., Ramirez-Castellanos J., González-Calbet J., "Influence of Sn and Cr Doping on Morphology and Luminescence of Thermally Grown $\mathrm{Ga}_{2} \mathrm{O}_{3}$ Nanowires" J. Phys. Chem. C. 117, 3036-3045 (2013).

[5] Nogales E., Hidalgo P., Lorenz K., Méndez B., Piqueras J., Alves E., "Cathodoluminescence of rare-earth implanted $\mathrm{Ga}_{2} \mathrm{O}_{3}$ and $\mathrm{GeO}_{2}$ nanostructures" Nanotechnol., 22, 285706 (2011).

[6] Hidalgo P., López A., Méndez B., and Piqueras J., "Synthesis and Optical Properties of $\mathrm{Zn}_{2} \mathrm{GeO}_{4}$ Microrods" Acta Materialia, 104, 84-90 (2016).

[7] Maestre D., Cremades A., Piqueras J., "Study of the morphology and luminescence of $\mathrm{SnO}_{2}$ micro- and nanostructures synthesized by a two-step thermal process" J. Cryst. Growth, 311, 1212-1216 (2009).

[8] Vila M., Díaz-Guerra C., Piqueras J., López-Conesa L., Estradé S., Peiró F., "Growth, Structure and mechanical resonance of $\mathrm{Bi}_{2} \mathrm{O}_{3}$ nano and microwires" CrystEngComm. 17, 132 (2015).

[9] Martínez-Criado G., Segura-Ruiz J., Chu M.-H., Tucoulou R., López I., Nogales E., Méndez B., and Piqueras J., "Crossed $\mathrm{Ga}_{2} \mathrm{O}_{3} / \mathrm{SnO}_{2}$ Multiwire Architecture: A Local Structure Study with Nanometer Resolution" Nano Letters, 14, 5479-5487 (2014).

[10] Alonso-Orts M., Sánchez A.M., Hindmarsh S.A., López I., Nogales E., Piqueras J., Méndez B., "Shape Engineering Driven by Selective Growth of $\mathrm{SnO}_{2}$ on Doped $\mathrm{Ga}_{2} \mathrm{O}_{3}$ Nanowires" Nano Letters, 17, 515-522 (2017).

[11] Cebriano T., Méndez B., Piqueras J., " $\mathrm{Sb}_{2} \mathrm{O}_{3}$ microrods: self-assembly phenomena, luminescence and phase transition" J. Nanopart Res, 15, 1667 (2013).

[12] Nogales E., García J. A., Méndez B., Piqueras J., "Red luminescence of $\mathrm{Cr}$ doped $\mathrm{Ga}_{2} \mathrm{O}_{3}$ nanowires" J. Appl. Phys. 101, 033517 (2007).

[13] Drouin D., Couture A.R., Joly D., Tastet X., Aimez V., Gauvin R., "CASINO V2.42—A Fast and Easy-to-use Modeling Tool for Scanning Electron Microscopy and Microanalysis Users" Scanning 29, 92 (2007).

[14] Binet L., Gourier D., "Origin of the blue luminescence of $\beta-\mathrm{Ga}_{2} \mathrm{O}_{3}$ " J. Phys. Chem. Solids, 59, 1241 (1998).

[15] Shimamura K., Víllora E. G., Ujiie T., Aoki K., "Excitation and photoluminescence of pure and Si-doped $\beta$ $\mathrm{Ga}_{2} \mathrm{O}_{3}$ single crystals" Appl. Phys. Lett. 92, 201914 (2008).

[16] Maestre D., Cremades A., Piqueras J., "Growth and luminescence properties of micro- and nanotubes in sintered tin oxide", J. Appl. Phys. 97, 044316 (2005).

[17] Das, S., Kar, S., Chaudhuri, S., "Optical properties of $\mathrm{SnO}_{2}$ nanoparticles and nanorods synthesized by solvothermal process" J. Appl. Phys. 99, 114303 (2006). 